\title{
Detection of small crustal deformation caused by slow slip events in southwest Japan using GNSS and tremor data
}

\author{
Megumi Fujita ${ }^{1 *}$, Takuya Nishimura ${ }^{2}$ and Shin'ichi Miyazaki ${ }^{3}$
}

\begin{abstract}
We stacked daily Global Navigation Satellite System (GNSS) displacement increments in southwest Japan to detect the cumulative crustal deformation accompanying non-volcanic low-frequency tremors from April 1, 2004 to December 31,2009, which are difficult to identify for individual slow slip events (SSEs). We successfully detected the crustal deformation associated with the tectonic stress releases coincident with tremor activity, which was larger than that caused by short-term SSEs detected in previous studies. To investigate the characteristics of the detected crustal deformation, we estimated the parameters of a rectangular fault model in segments along the Nankai Trough using a non-linear inversion method. Consequently, we obtained results that were concordant with those of previous studies and positive linear correlations between the moment and tremor energy in some segments. The linear regression slopes differed, reflecting the fact that the excitation efficiency of the tremors resulting from slow slip was different for each segment. Our results suggest that tremor activity serves as a proxy for SSES, reflecting the different characteristics of SSEs in segments.
\end{abstract}

Keywords: Slow slip event, Tremor, GNSS, Southwest Japan

\section{Introduction}

Megathrust earthquakes occur repeatedly with certain recurrence intervals along the boundary between a subducting oceanic plate and continental plate. "Slow earthquakes," with fault slip rates between the fast rupture of a regular earthquake and stable sliding, have also been detected by dense geodetic and seismological observation. Slow slip events (SSEs), which constitute one type of slow earthquake, release accumulated inter-plate strain with durations of days to years and radiate no seismic energy (cf. Schwartz and Rokosky 2007). SSEs often occur at down-dip extensions of the megathrust seismogenic zones on plate interfaces and perturb the surrounding stress field. Although SSEs release accumulated strain in SSE zones, they can increase the strain in up-dip seismogenic locked zones. Therefore, better understanding

\footnotetext{
${ }^{*}$ Correspondence: fujita.megumi.65s@st.kyoto-u.ac.jp

${ }^{1}$ Graduate School of Science, Kyoto University, Gokasho, Uji,

Kyoto 611-0011, Japan

Full list of author information is available at the end of the article
}

of SSEs is very important for improving earthquake hazard assessment (e.g., Obara and Kato 2016).

In southwest Japan, the Philippine Sea Plate subducts beneath the Amurian Plate along the Nankai Trough (Fig. 1) and many previous studies have indicated that various types of slow earthquakes have occurred along the plate interface. Long-term SSEs with durations of several months to years have been observed using the Global Navigation Satellite System (GNSS) from the GNSS Earth Observation Network System (GEONET) established by the Geospatial Information Authority of Japan (GSI) in the Bungo channel (e.g., Hirose et al. 1999), and the moment was estimated to be approximately equivalent to moment magnitude $(\mathrm{Mw}) 7$ in each event. In western Shikoku, smaller long-term SSEs with durations of 1-2 years have also been detected (Takagi et al. 2016). Short-term SSEs with durations of several days to weeks have been observed using tilt data from the National Research Institute for Earth Science and Disaster Resilience (NIED) Hi-net (e.g., Obara et al. 2004) and, in recent years, GNSS data (Nishimura et al. 


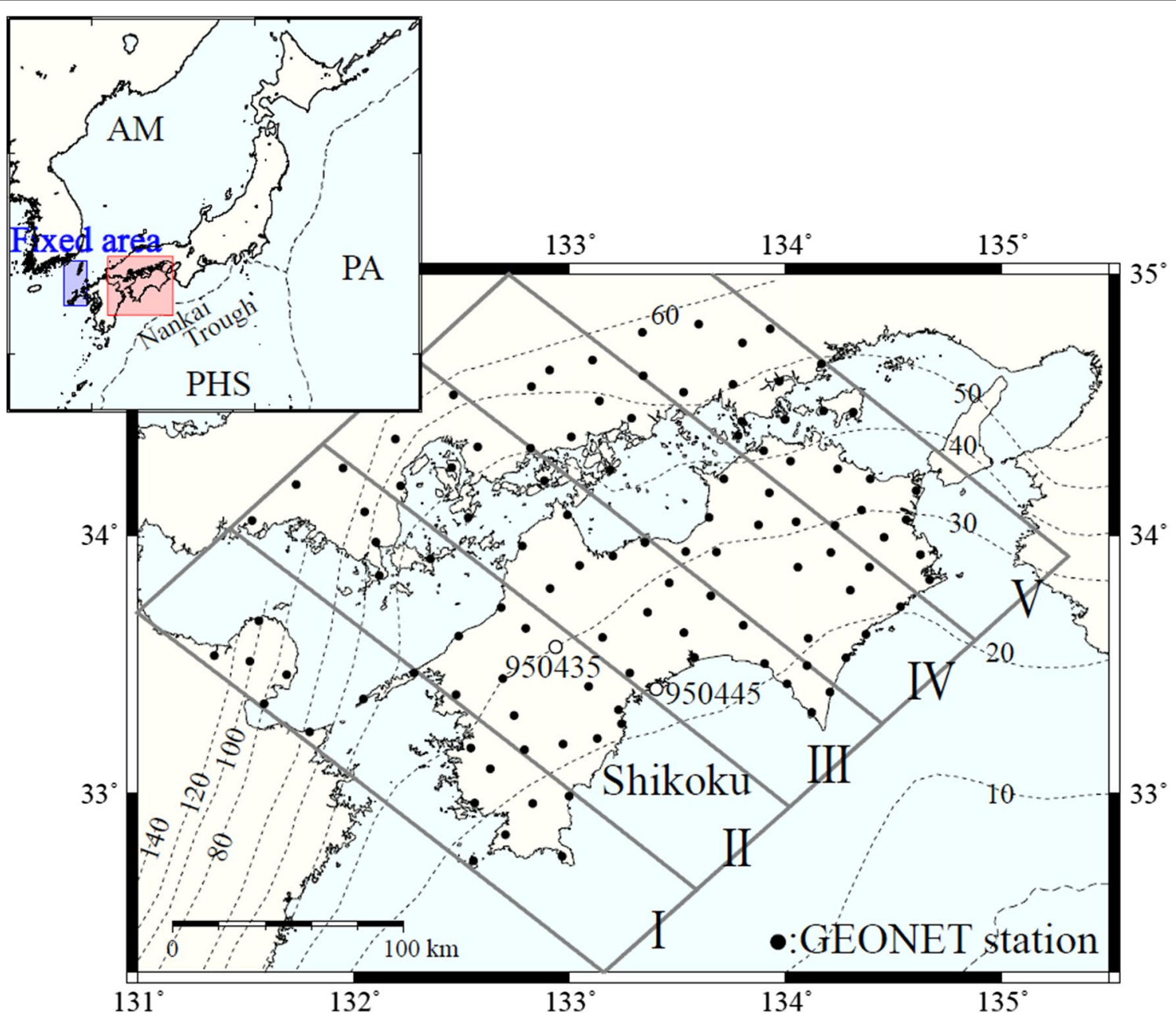

Fig. 1 Distribution of GNSS stations used in this study. Black circles indicate GEONET stations and open circles indicate GEONET stations 950435 and 950445 whose GNSS time series are plotted in Fig. 2. Black dashed contours show the iso-depth of the plate interface between the Philippine Sea and Amurian Plates (Baba et al. 2002; Nakajima and Hasegawa 2007; Hirose et al. 2008). Gray rectangles indicate the boundaries of five segments. (Inset) Tectonic map around the Japanese Islands. PA, PHS, and AM denote the Pacific, Philippine Sea, and Amurian Plates, respectively. Red and blue rectangles indicate the analysis area in this study and the area where the six reference GNSS stations are located, respectively

2013; Nishimura 2014). The estimated moment magnitudes of short-term SSEs range from 5.4 to 6.2 (Sekine et al. 2010), which are smaller than those of long-term SSEs. In particular, short-term SSEs in southwest Japan, which are synchronized with non-volcanic low-frequency tremor activity (Obara 2002), have repeatedly occurred with a recurrence interval of about 6 months (Hirose and Obara 2005). Such coincidental occurrence of SSEs and tremors is called Episodic Tremor and Slip (ETS) (Rogers and Dragert 2003). It is reported that there is a strong correlation between SSE moment and tremor energy and it, therefore, suggests that tremor activity can be a proxy for short-term SSEs in southwest Japan (Hiramatsu et al. 2008).
Frank (2016) focused on a rather quiet period with minor tremor activity and no major slow slip events detected geodetically and calculated the cumulative GNSS displacement by summing up the displacement increments during minor tremor events. He reported that small but significant crustal deformation releasing accumulated strain could be detected by stacking the GNSS displacement increments because the signal-tonoise ratio of GNSS time series can be increased by calculating cumulative displacements.

Slow slip events in southwest Japan generally have smaller magnitudes than those in Cascadia, and it is more difficult to detect their signals. As mentioned above, some such events have been observed using tiltmeters 
and GNSS. However, it is not always straightforward to identify the signals of each SSE in GNSS data because they are small and buried in noise. Furthermore, there may be some crustal deformation accompanying minor tremor episodes that are undetectable even using tiltmeters because their signals are too weak to detect geodetically (Sekine et al. 2010). Therefore, in this study, we applied the stacking method proposed by Frank (2016) to GNSS and tremor data in southwest Japan to detect the cumulative displacements associated with tectonic stress release events, including previously identified SSEs, which are difficult to identify for each individual SSE in GNSS data. In addition, we estimated the fault parameters based on the detected surface displacements and analyzed the different characteristics of the SSEs, including the relation between the moment of the SSE and the energy of the tremor activity in each segment.

\section{Data and methods}

\section{Data and pre-processing of GNSS time series}

We selected 111 GNSS stations around Shikoku in southwest Japan (Fig. 1) and used their daily coordinates estimated by employing the precise point positioning (PPP) strategy using GIPSY software between April 1, 2004 and December 31, 2009. The analysis period is limited by the beginning of the tremor catalog studied by Annoura et al. (2016), as mentioned later. The last day of our analysis, that is, December 31, 2009 is selected to avoid any possible effects of the long-term SSEs in the Bungo channel (e.g., Ozawa 2017) and co- and post-seismic displacement of the 2011 Tohoku-oki earthquake (e.g., Nishimura et al. 2011). This period includes some short-term SSEs detected from tilt data (Sekine et al. 2010) and GNSS data (Nishimura et al. 2013). We divided the analysis area into five segments labeled I to V along the Nankai Trough. The long sides of the segments were almost parallel to the direction of plate convergence and each segment was analyzed separately because small SSEs associated with minor tremor activity below one segment are expected to generate enough small surface displacements to ignore in the neighboring segments. As tremor data, we used the catalog studied by Annoura et al. (2016) and calculated the daily sum of the tremor seismic energy in each segment.

We defined the displacement increments of the GNSS time series as shown in Eq. (1):

$$
\Delta x(d)=x(d+1)-x(d),
$$

where $x$ denotes a daily coordinate component [eastwest (EW) or north-south (NS)] and $d$ denotes the successive number of GNSS coordinates during the analysis period. When there were missing GNSS coordinates on some day, we ignored them and counted only the number of days with coordinates existing at each GNSS station. Specifically, $d$ was defined as shown in Eq. (2):

$$
d=[1 \cdots N]
$$

where $N$ is the total number of days with GNSS coordinates existing at each GNSS station during the analysis period. Note that $d$ does not indicate time in years $t$, which is described later in Eq. (4).

As pre-processing of the GNSS time series, we firstly removed the coseismic and artificial offsets. As discussed in detail later, we focused on the displacement increments of the GNSS time series defined above in Eq. (1). Therefore, we eliminated the displacement increments for 3 days before and after major earthquakes as well as antenna changes based on the list provided by the GSI. We selected these major earthquakes based on the following criteria. Firstly, we obtained the list of earthquakes that caused crustal deformation at GNSS stations compiled by the GSI (http://mekira.gsi.go.jp/catalogue/index.html). Then, we calculated the hypocentral radius $R_{0}$ of an area suffering coseismic deformation by applying Eq. (3) (Okada 1995) for every earthquake listed by the GSI during our analysis period:

$$
\log U_{\max }=1.5 M-2 \log R_{0}-6.0,
$$

where $U_{\max }$ denotes the expected maximum coseismic crustal displacement in centimeters, $M$ denotes the magnitude of the earthquake, and $R_{0}$ denotes the hypocentral radius in kilometers. In this study, we used $0.05 \mathrm{~cm}$ for $U_{\max }$. Then, we calculated the epicentral radius $R$ from $R_{0}$ and the earthquake depth. Using the GNSS stations within in the radius $R$, we identified the major earthquakes and eliminated the displacement increments for 6 days.

Secondly, we calculated the relative displacements with respect to the average time series of six GNSS stations (station numbers 950456, 950457, 950458, 950462, 960691, and 960692) to remove common mode errors and to extract the GNSS time series with the Amurian Plate fixed. Stacking the time series of multiple reference stations reduces the effects of the scatter noise of the reference stations (Takagi et al. 2016; Wdowinski et al. 1997).

Thirdly, we estimated the annual and semiannual components using a linear least squares method and subtracted them from the GNSS time series, using Eq. (4):

$$
\begin{aligned}
x^{\prime}(t) & =a+b t+c_{1} \sin (2 \pi \omega t)+c_{2} \cos (2 \pi \omega t) \\
& +c_{3} \sin (4 \pi \omega t)+c_{4} \cos (4 \pi \omega t),
\end{aligned}
$$

where $x^{\prime}(t)$ is the best fit model resulting from the linear regression, $t$ denotes the time in years, and $\omega$ is the angular frequency ( $1 \mathrm{rad} /$ year in this case). $a, b, c_{1}, c_{2}, c_{3}$, and $c_{4}$ are the parameters estimated using the linear least squares method. Then, we calculated the standard error $s$ expressed in Eq. (5) and removed the daily coordinate, 
$x(t)$, if $\left|x(t)-x^{\prime}(t)\right|>2 s$ to eliminate the outliers from the GNSS time series:

$$
s=\sqrt{\frac{\sum_{N}\left(x(t)-x^{\prime}(t)\right)^{2}}{N-2}} .
$$

Fourthly, we estimated the loading velocities due to inter-plate locking and removed the effects of the stress loading in the locked up-dip regions of the SSE zones. We extracted the displacement increments only for periods during which there was no tremor energy in one segment. Then, we cumulatively summed up these increments, estimated the loading velocities using a least squares method, and subtracted the estimated loading velocities from the GNSS time series. That means we assumed that the area including the SSE zones and their up-dip zones was locked during periods of no tremor energy, which was considered to be the reference state in our analysis. That is, the stacked displacements during tremor activity were the perturbations from the average velocity during no tremor activity and can be considered to be the total signals of small tectonic stress release.

\section{Extraction and stacking of displacement increments during release period}

We focused on the crustal deformation accompanying tremor activity and extracted the displacement increments during tremor activity following the stacking method proposed by Frank (2016). After pre-processing the GNSS time series, we calculated the displacement increments by applying Eq. (1) again. Next, we extracted the increments for the release period according to the daily sum of tremor energy using the catalog studied by Annoura et al. (2016). Here, we converted the time in the tremor catalog from Japan Standard Time (JST) into Coordinated Universal Time (UTC), which is approximately equal to GPS time. If the daily sum of tremor energy was above a certain threshold, we categorized the displacement increments into the release period. In other words, we considered displacements due to the stress release to occur only on days of high tremor energy. Otherwise, we considered there to be no displacement at the GNSS stations, which we called the stable period. We changed the tremor energy threshold from $0.05 \times 10^{7} \mathrm{~J}$ to $3.00 \times 10^{7} \mathrm{~J}$ in increments of $0.05 \times 10^{7} \mathrm{~J}$.

Then, we cumulatively summed up the displacement increments for the release period (Fig. 2). In Fig. 2a-d, the GNSS time series have been rearranged to separate the stable period (red lines) from the release period (blue lines). The GNSS time series before rearrangement are shown in Additional file 1: Figure S1. By cumulatively summing the increments of the release period, the signalto-noise ratio during tremor activity can be increased and the cumulative displacement can be regarded as the total displacement due to the tectonic stress release, that is, the total signal of small SSEs. Finally, we estimated the release velocities based on the cumulative displacements for the release period using a least squares method. We also estimated the standard error of the linear regression slope $\sigma$ using Eq. (6):

$$
\sigma=\frac{s}{\sqrt{\sum_{N}(x-\bar{x})^{2}}},
$$

where $\bar{x}$ is the mean of $x$.

\section{Results}

The calculated release velocities per day, especially on the up-dip side of the tremor activity, are generally oriented opposite to the direction of plate convergence (Fig. 3a-c). In segment $\mathrm{V}$, the number of days categorized into the release period was very small because of little tremor activity (Fig. 4e), resulting in large scatter of the release velocities. Therefore, only the results in segments I to IV are plotted in Fig. 3, although the results obtained in all segments with a threshold of $0.05 \times 10^{7} \mathrm{~J}$ are provided in Additional file 1: Figure S2. As shown in Fig. 3a-c, the higher the tremor energy threshold, the greater the estimated release velocities, because they were extracted only for greater tectonic stress release coincident with tremor activity. Therefore, we succeeded in detecting small crustal deformation accompanying tremor activity. However, when the threshold is too high, the release velocities exhibit considerable scatter and larger error ellipses (Fig. 3d). This is probably because the number of days categorized into the release period decreases as the threshold increases (Fig. 4) and the signal-to-noise ratio of the release period becomes worse. It is expected that by increasing the analysis period and the number of days categorized into the release period, the signal-tonoise ratio of the release period could be improved and the release velocities could be observed even with a high threshold.

\section{Discussion}

\section{Cumulative displacements and durations for the release period}

To clarify the performance of the stacking method, we compared our stacked displacements with the cumulative displacements predicted by the fault model of the previously identified short-term SSEs. We used the elastic dislocation model in a homogeneous half-space medium (Okada 1992) to calculate the displacements. The short-term SSE models were estimated using tilt data from the NIED Hi-net (e.g., Sekine et al. 2010) and the GNSS data employed by Nishimura et al. (2013). 

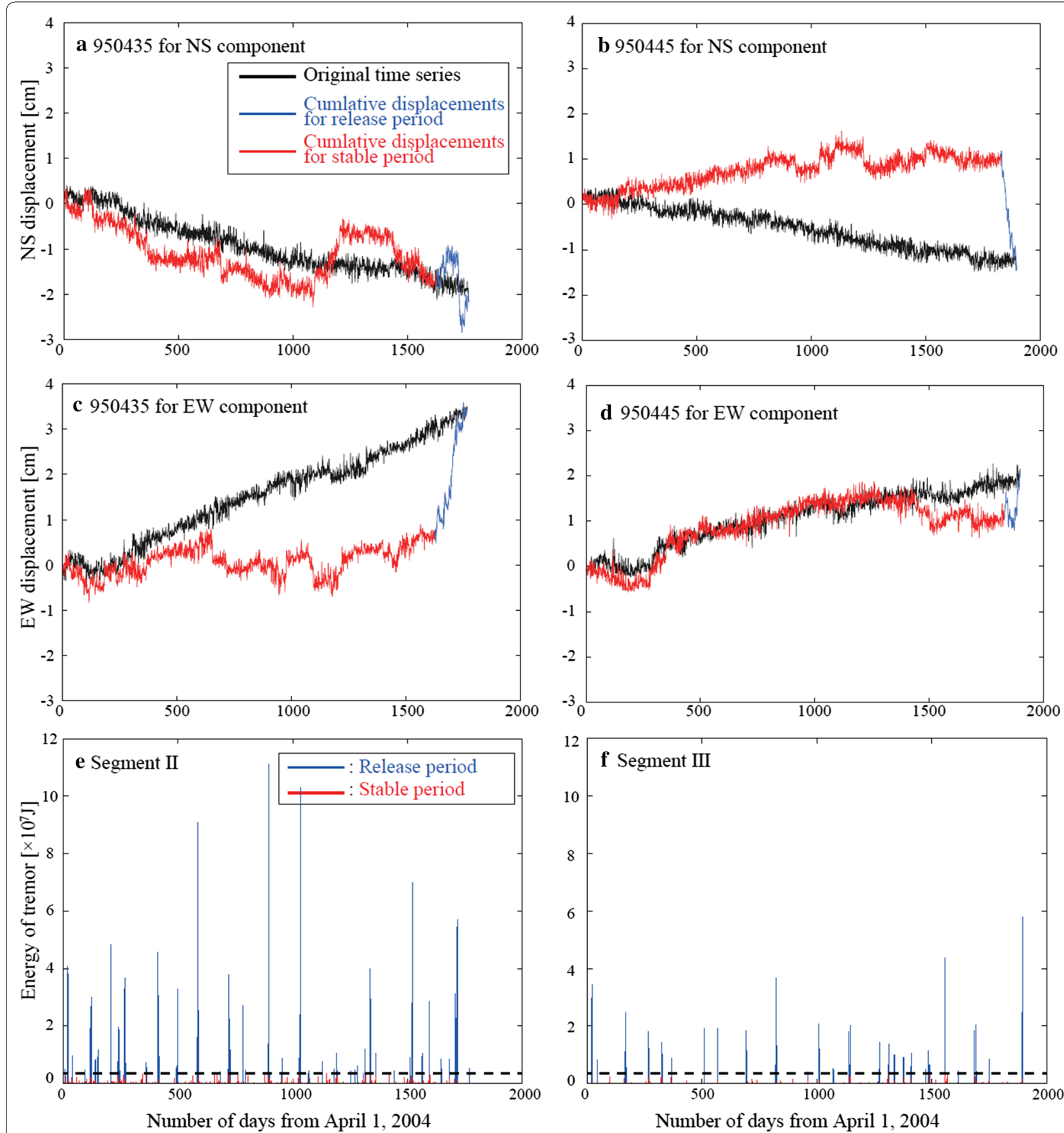

Fig. 2 Rearranged GNSS time series and the time series of the daily sum of tremor energy between 2004 and 2009 with a tremor energy threshold of $0.35 \times 10^{\prime} \mathrm{J}$. a , b GNSS time series for NS component at station 950435 in segment II and station 950445 in segment III, respectively. c, d Same as (a) and (b), but for EW component. Black, red, and blue traces show the corrected GNSS time series, cumulative surface displacements for the stable period, and cumulative surface displacements for the release period, respectively. e, f Daily sum of tremor energy in segments II and III, respectively. Black horizontal dashed lines show the threshold of $0.35 \times 10^{7} \mathrm{~J}$. Red and blue vertical bars correspond to the groups for the stable and release periods, respectively

We used the rectangular fault model parameters estimated in those previous studies in our analysis period (i.e., between April 1, 2004 and December 31, 2009) and calculated the cumulative displacements. For our analysis period, the fault models of 24 events were estimated by the NIED (and were collected in what is 

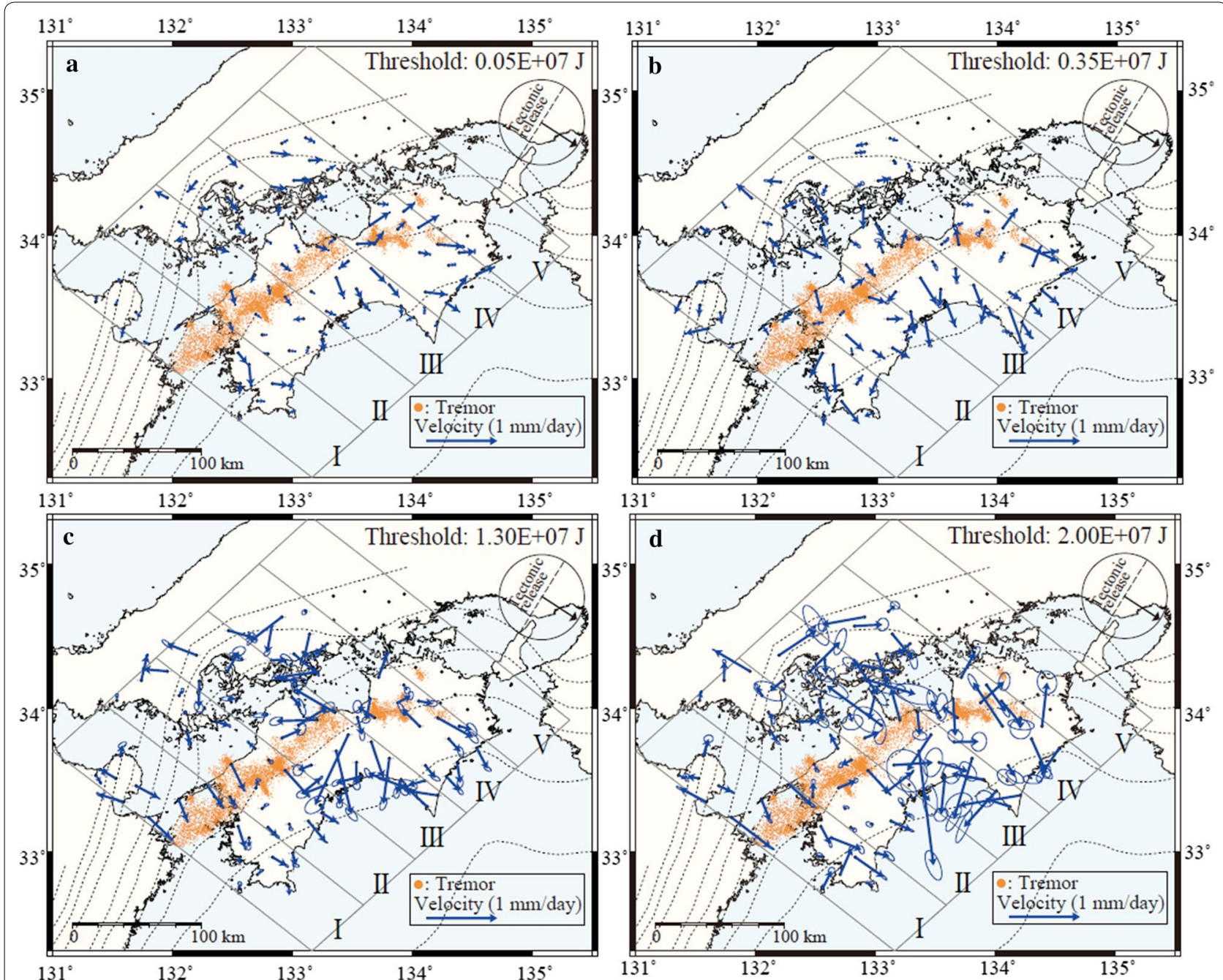

Fig. 3 Examples of the release velocity patterns with various thresholds between April 1, 2004 and December 31, 2009. Blue arrows indicate the estimated release velocities per day. Error ellipses at arrow heads show one standard error. Orange dots indicate the epicenters of non-volcanic tremors (Annoura et al. 2016) during our analysis period. Black arrows in circles are the expected directions of tectonic stress release, which is opposite to the direction of the subducting Philippine Sea Plate based on MORVEL (DeMets et al. 2010). a Release velocities with a tremor energy threshold of $0.05 \times 10^{7} \mathrm{~J}$. b Same as $\mathbf{a}$, but with a tremor energy threshold of $0.35 \times 10^{7} \mathrm{~J}$. c Same as $\mathbf{a}$, but with a tremor energy threshold of $1.30 \times 10^{\prime} \mathrm{J}$. d Same as a, but with a tremor energy threshold of $2.00 \times 10^{\prime} \mathrm{J}$

hereinafter called the NIED catalog), which consists of 14 events from April 2004 to March 2008 listed by Sekine et al. (2010) and 10 events from April 2008 to December 2009 reported by the NIED (2009a, b, 2010a, b). We also used the fault models of 35 events along the Nankai Trough, estimated by Nishimura et al. (2013).

Figure 5 compares the cumulative displacements calculated from our results and those of the previous studies. The cumulative displacements for the release period were calculated approximately from the products of the estimated release velocities and the number of days categorized into the release period, assuming that the estimated velocities were constant during the release period.
Figure 5 suggests that the cumulative displacements estimated in this study are generally larger than those for the SSEs detected previously, indicating that we succeeded in detecting more SSEs than those included in the previous SSE catalogs. In particular, our stacked displacements coincident with tremor activity in segment IV are much larger than those predicted by the models developed using tilt data (Fig. 5a). In other words, we were able to detect more SSEs, including smaller ones that are difficult to identify individually.

As described in "Results", the higher the tremor energy threshold, the greater the estimated release velocities, but the smaller the cumulative displacements (Fig. 5b-d), because as the threshold increases, the number of days 

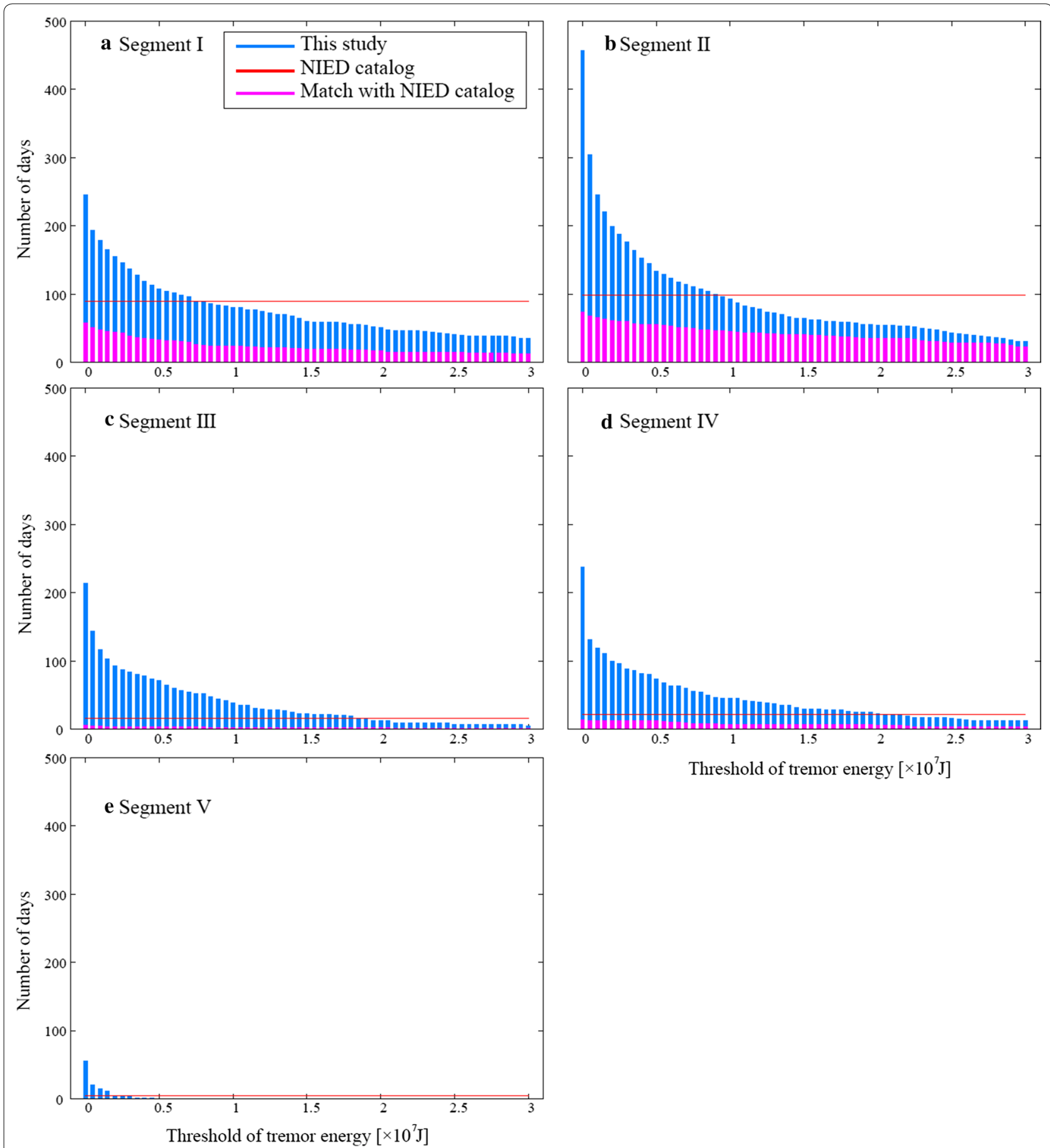

Threshold of tremor energy $\left[\times 10^{7} \mathrm{~J}\right]$

Fig. 4 Comparison of the duration and timing of SSEs in our study with those in the NIED catalog in each segment. Blue vertical bars show the number of days categorized into the release period when the tremor energy threshold is changed. Pink vertical bars show the number of days in which the SSE periods in this study correspond to those in the NIED catalog. Red horizontal lines show the total durations of SSEs in the NIED catalog. a Comparison in segment I. b Same as $\mathbf{a}$, but in segment II. c Same as $\mathbf{a}$, but in segment III. $\mathbf{d}$ Same as $\mathbf{a}$, but in segment IV. e Same as $\mathbf{a}$, but in segment $\mathrm{V}$ 

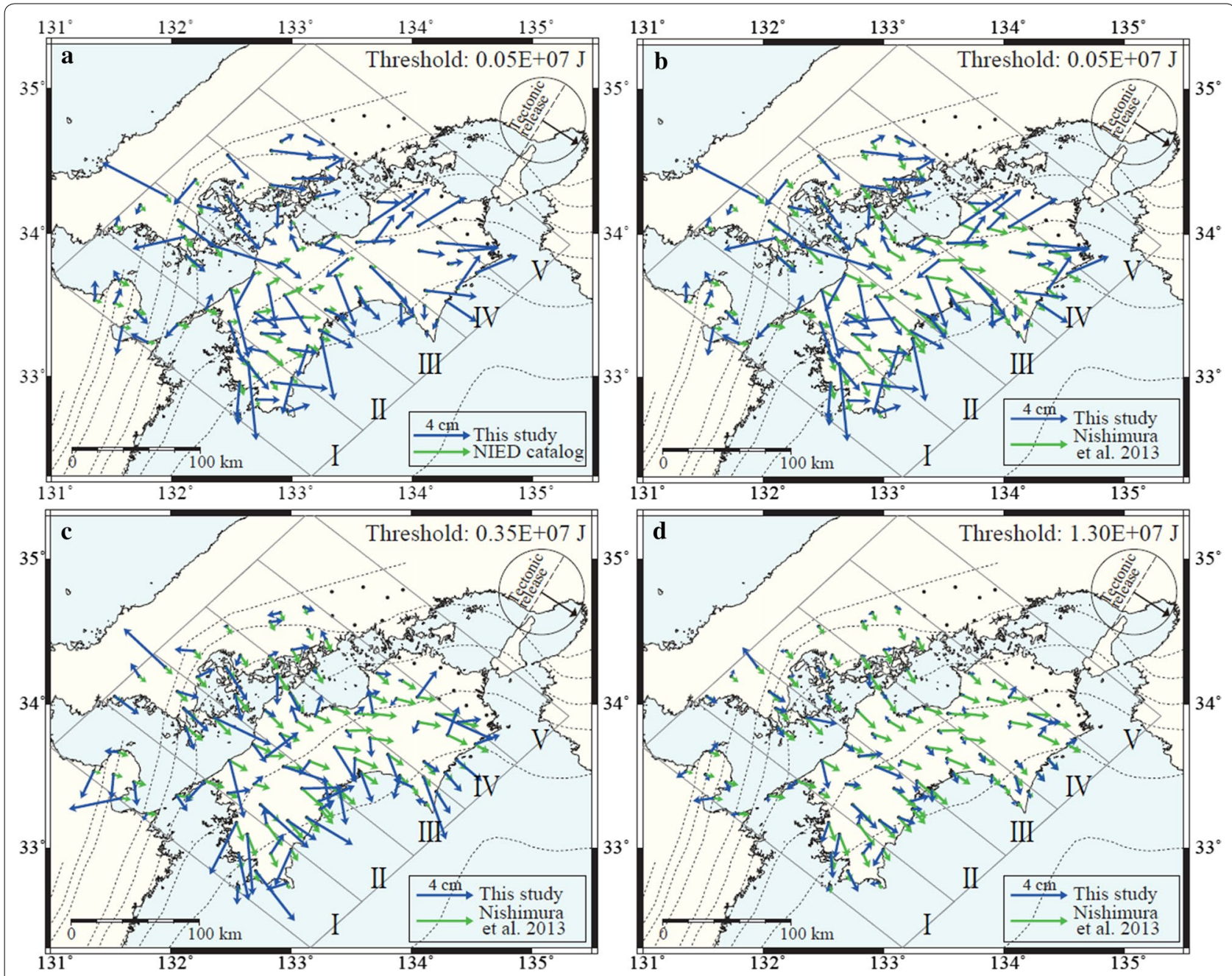

Fig. 5 Comparison of the cumulative displacements between our stacking method and the previous SSE models from April 1, 2004 to December 31, 2009. Blue and green arrows indicate the cumulative displacements obtained in our analysis and those calculated from the previous SSE models, respectively. a Model of the NIED catalog with a tremor energy threshold of $0.05 \times 10^{7} \mathbf{~ J}$. b Same as $\mathbf{a}$, but with the models of Nishimura et al. (2013). c Same as $\mathbf{b}$, but with a tremor energy threshold of $0.35 \times 10^{7} \mathrm{~J}$. d Same as $\mathbf{b}$, but with a tremor energy threshold of $1.30 \times 10^{7} \mathrm{~J}$

categorized into the release period decreases. That is, the number of days categorized into the release period affects the cumulative displacements more than the release velocity.

In segments III and IV, the cumulative displacements estimated in this study are much smaller when the threshold is $1.30 \times 10^{7} \mathrm{~J}$ (Fig. 5d) than when it is $0.05 \times 10^{7} \mathrm{~J}$ (Fig. 5b), which means that crustal deformation corresponding to relatively intense tremor activity did not occur in these segments. In addition, the amplitude of the cumulative displacements estimated in this study is roughly similar in segments I, II, and III when the threshold is $0.35 \times 10^{7} \mathrm{~J}$ (Fig. 5c). However, when the threshold is $1.30 \times 10^{7} \mathrm{~J}$ (Fig. 5d), the amplitude in segment III is much smaller than those in segments I and II, and the latter amplitudes are approximately equal to that of the cumulative displacements of SSEs detected previously. Taking into account these contrasts, it could be suggested that the excitation efficiency of tremors due to slow slip is different for each segment in western Shikoku.

Furthermore, we compared the duration and timing of the release period in this study and those in the previous SSE catalogs. Nishimura et al. (2013) estimated only the middle dates of SSE episodes and did not estimate the durations of SSEs. Therefore, we compared the durations only with the NIED catalog, according to the following procedure. We made the space-time plot of short-term SSEs in the NIED catalog, projecting in the strike direction of $N 38^{\circ}$ E (Additional file 1: Figure S3). We counted durations of SSEs every segment when a part of SSE along strike is included in the segment. Figure 4 shows the comparison of the total duration of SSEs in 
this study with that in the NIED catalog. In segments I to IV, the number of the days for the release periods, which are included in SSE durations of the NIED catalog, is smaller than the total duration of SSEs in the NIED catalog at any tremor energy thresholds. This result might imply that the short-term SSEs detected by the NIED were composed of intermittent slow slips accompanying tremor activity and that the actual duration of slow slip was shorter than that of SSEs identified in the previous study, similar to the long-term SSEs in Guerrero reported by Frank et al. (2018). Our method might be effective in extracting the real active period in which short-term SSEs accompanying tremor activity occurs in more detail. We also made the space-time plot of short-term SSEs estimated by Nishimura et al. (2013) and confirmed whether the middle dates of SSE episodes were included in the release period in our study (Additional file 1: Figure S4). Consequently, The middle dates of 30 out of 33 models in segments I to IV estimated by Nishimura et al. (2013) were included in the release period in our study with a tremor energy threshold of $0.05 \times 10^{7} \mathrm{~J}$. Our analysis quantitatively shows the synchronization of SSEs with tremor activity, which Nishimura et al. (2013) also suggested. It also suggests that many strain release episodes accompanying with tremors may be missed in the catalogs of the previous studies.

\section{Different characteristics of the estimated fault and tremor energy in each segment}

To analyze the different characteristics in each segment, we estimated the source region of the detected small crustal deformation using a rectangular fault model. The input data of the source estimation were the estimated release velocities per day such as Fig. 3 with varying the tremor energy threshold every segment. We used a nonlinear inversion method (Matsu'ura and Hasegawa 1987) and a homogeneous elastic half-space model (Okada 1992) to estimate the fault parameters. The initial locations of the center of the rectangular fault model were the centers of the stations in each segment. The initial width, rake, and slip velocity were the averages of the estimated parameters listed in Table 1 of Sekine et al. (2010) for segments I, II, and III and those listed in Table 2 of Sekine et al. (2010) for segment IV. The initial slip velocity is derived from the average slip velocity for all the shortterm SSEs in Sekine et al. (2010) which are included in our analysis period. The depth, strike, and dip were constrained to fit the iso-depth contours of the Philippine Sea Plate (Baba et al. 2002; Nakajima and Hasegawa 2007; Hirose et al. 2008). Because the coverage of the GNSS stations along the strike was limited in each segment, the length of the rectangular fault was difficult to estimate. Therefore, we fixed the length to $1000 \mathrm{~km}$, approximating an infinitely long fault along the strike. Finally, we estimated five parameters: longitude, latitude, width, rake, and slip velocity.

Estimated rectangular fault models for different tremor energy thresholds show wide ranges of widths and rakes, and some of them are unrealistic due to the low signal-tonoise ratio of the release velocity, especially in segment IV. Therefore, in this paper, we discuss only some common characteristics of the well-fitted fault model defined later in segment I, II, and III. Figure 6 shows the examples of the estimated rectangular fault models in segments I, II, and III, respectively.

In these segments, the estimated faults are located above the tremor activity and these are spatially concordant with those of previous studies. The average slip velocity of well-fitted models (Additional file 1: Figures S5-S7) are $3.4 \mathrm{~mm} /$ day, $9.2 \mathrm{~mm} /$ day, and $2.6 \mathrm{~mm} /$ day in segment I, II, and III, respectively. Although the average slip velocity in segment II is faster, it is probably artificial because there was a strong trade-off between the area of the rectangular fault and the average slip and they couldn't be well resolved (Additional file 1: Figure S6). However, the average slip velocities in segment I and III are approximately equal to that of short-term SSEs estimated in western Shikoku by the NIED (e.g., $2.9 \mathrm{~mm}$ /day which is an average calculated by dividing the slip by the duration).

As described in "Introduction", the previous studies reported a strong correlation between tremor energy and SSE moment. Hiramatsu et al. (2008) analyzed SSEs in the Tokai region, about $500 \mathrm{~km}$ east of the western Shikoku region along the Nankai Trough and reported that there were positive correlations between the seismic moments of the SSEs and the apparent moments of the tremors. To confirm whether there were these characteristics between segments in our results, we plotted the relation between the daily moment derived from the estimated slip velocity and the area of the rectangular fault model and the average of daily tremor energy during the release period when we changed the tremor energy threshold in Additional file 1: Figure S8. As mentioned above, when we estimated the SSE fault models, we fixed the length to $1000 \mathrm{~km}$, approximating an infinitely long fault along the strike. Therefore, we normalized the moment by the length and calculated the moment per length. From all tremor energy and moment plots (Additional file 1: Figure S8), we could not identify any relationship between the SSE moment and average tremor energy, probably due to the low signal-to-noise ratio of the release velocities, as mentioned above, and many unrealistic estimated SSE models are also present in Additional file 1: Figure S8. To find the correlation between estimated SSE moments and tremor energy, 


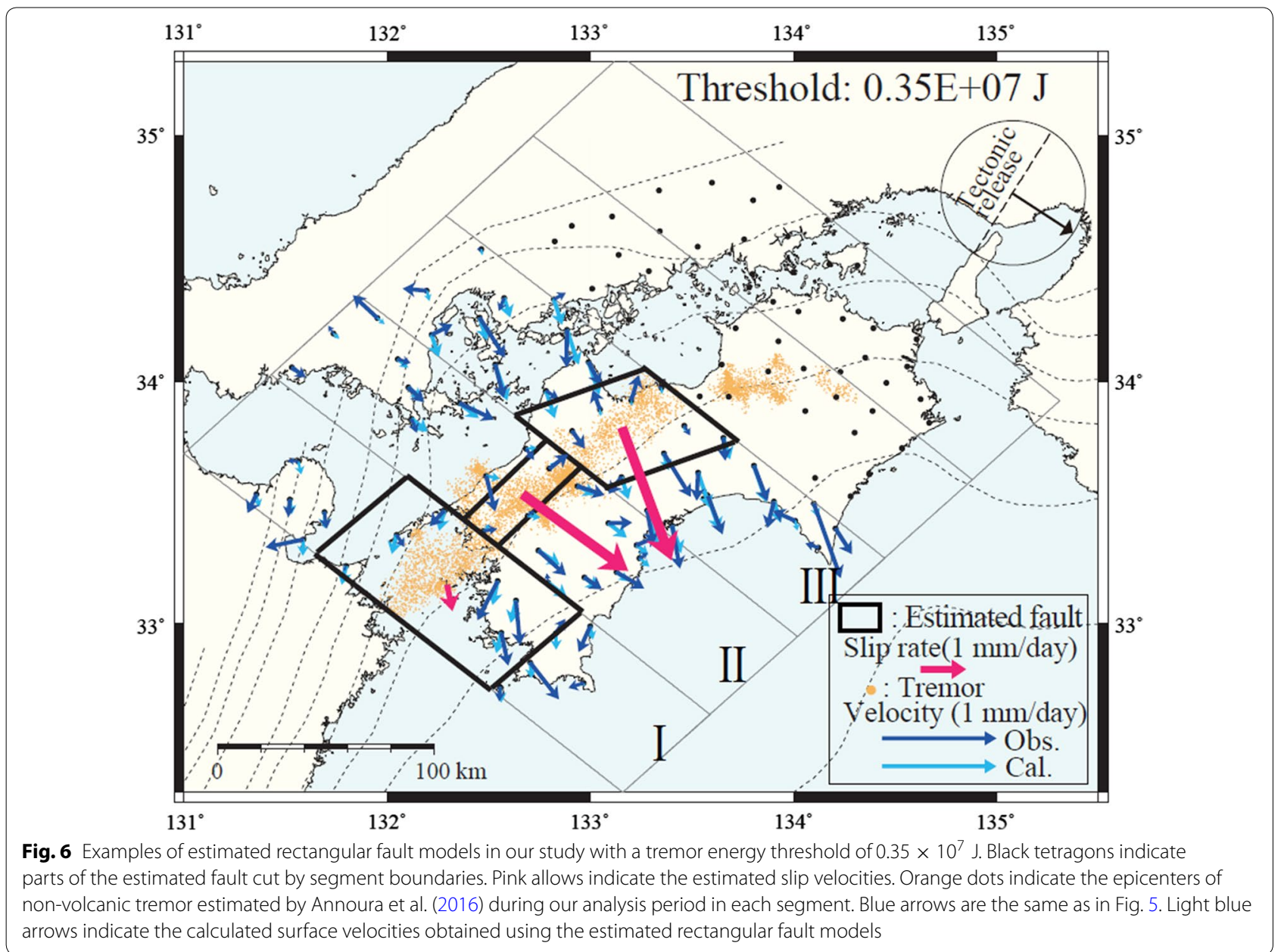

we chose 'well-fitted models' of the estimated rectangular fault models based on the following criteria (Fig. 7). Firstly, we focused only on the estimated models with a tremor energy threshold of $1.50 \times 10^{7} \mathrm{~J}$ or less. This is because the signal to noise ratio of the release period is better with a smaller threshold and we could obtain the rather robust models, although it becomes worse with a too large threshold as described in "Results". Secondly, we calculated the correlation coefficients between estimated SSE moment and average of tremor energy increasing the number of data used in the calculation in descending order of residual reductions in the fault estimation in each segment (Additional file 1: Figure S9). We stopped to increase the number of data when correlation coefficient was lower than 0.7. Based on these criteria, we chose 12, 9, and 11 models in segments I, II, and III, respectively, and call these fault models 'well-fitted models.' We obtained positive linear correlations between the moment and tremor energy in segments I, II, and III. Our results indicate there are similar relationships between the SSEs and tremors in western Shikoku with those

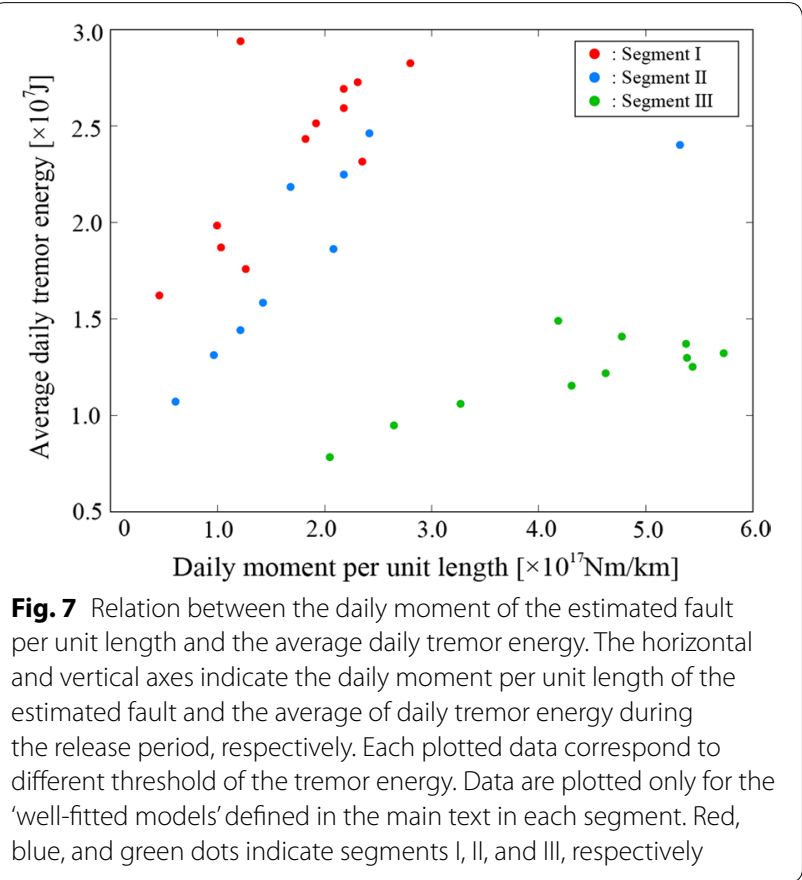


reported by Hiramatsu et al. (2008). In addition, these results indicate that the slow slip rate was high when the tremor became active in the corresponding segment. This feature is concordant with the characteristics of our results that the higher the tremor energy threshold, the greater the release velocities because the signals were extracted only for greater tectonic stress release coincident with tremor activity, as described in "Results". Furthermore, the linear regression slopes in segments I and II are steeper than that in segment III, which means that an SSE with the same moment activates more tremors in segments I and II than in segment III. Thus, the proportionality coefficients between the SSEs and the energy of the corresponding tremor activity are different in each segment. This characteristic is concordant with the results reported by Yabe and Ide (2014) that the energy rate of tremors in western Shikoku is larger than that in central Shikoku.

Some problems in the fault model estimation should be noted. We could not obtain a very robust model in each segment, as mentioned above and shown in Additional file 1: Figures S5-S7, and could not obtain such a correlation in segment IV either, probably due to the low signal-to-noise ratio in the release period. By analyzing a longer period and improving the signal-to-noise ratio by increasing the number of days categorized into the release period, the different characteristics of the SSEs in each segment could be analyzed more precisely. Despite the issues described above, the variations between segments I, II, and III obtained through our analysis suggest that the characteristics of SSEs differ by segment in western Shikoku.

\section{Conclusions}

Applying the staking method proposed by Frank (2016) to GNSS data enabled successful detection of the small crustal deformation accompanying tremor activity in southwest Japan. Comparison with the results of previous studies also indicated that we were able to detect larger crustal deformation than those caused by the previously detected SSEs.

The fault models estimated from the stacked displacements yielded results that were concordant with those of previous studies and linear relations between the moment and tremor energy in some segments. In addition, the obtained linear positive correlations suggest that large slow slip occurred when the active tremors were in the segments in western Shikoku (segments I and II) and central Shikoku (segment III). The differences in the linear regression slopes probably reflect the differences of the proportionality coefficients between the SSEs and corresponding tremor energy in this region. In this case, the proportionality coefficient in western Shikoku was found to be greater than that in central Shikoku. These results indicate that the characteristics of the locations where SSEs occur and the excitation efficiencies of the resulting tremors are different for each segment. These results might suggest that an average strength of tremor sources varies along the Nankai Trough even if tremor sources are distributed homogeneously. In other words, tremor activity could serve as a proxy for SSEs, reflecting the different characteristics of the SSEs in the segments along the Nankai Trough.

However, we could not obtain very stable fault models overall because the signal-to-noise ratio of the release period was low. By analyzing a longer period, it would be possible to describe the overall differences between the segments in southwest Japan more precisely and to clarify the characteristics of small SSEs in detail. Furthermore, this stacking method might be useful to detect small crustal deformation with tremor activity as cumulative displacements in other regions.

\section{Supplementary information}

Supplementary information accompanies this paper at https://doi. org/10.1186/s40623-019-1075-x.

Additional file 1: Figure S1. Daily tremor energy and GNSS time series. We use the same color in this figure as in Fig. 2. (a), (b) Daily sum of tremor energy in segment II and III, respectively. (c), (d) Displacement increments categorized into stable and release period for NS component at station 950435 in segment II and station 950445 in segment III, respectively. (e), (f) GNSS time series before rearrangement for NS component at station 950435 and station 950445, respectively. (g), (h) Same as (c) and (d), but for EW component. (i), (j) Same as (e) and (f), but for EW component. Figure S2. Release velocities between April 1, 2004 and December 31, 2009 with a tremor energy threshold of $0.05 \times 10^{7} \mathrm{~J}$ in all segments. We use the same symbols in this figure as in Fig. 3. Figure S3. Space-time plot of short-term SSEs along the Nankai Trough, in the NIED catalog during our analysis period. The vertical axis indicates the distance from the lower left point of segment I (Fig. 1) in the strike direction of N38 E. Gray horizontal lines show the boundaries of the segments. Pink lines show SSE faults in the NIED catalog. Figure S4. Space-time plot of short-term SSEs along the Nankai Trough, detected by Nishimura et al. (2013) during our analysis period. The vertical axis and gray horizontal lines are the same as in Figure S3. The horizontal axis indicates the middle days of SSE period. Pink lines show SSE faults estimated by Nishimura et al. (2013). Blue lines show the timing of release period in each segment with a tremor energy threshold of $0.05 \times 10^{7} \mathrm{~J}$. Figure S5. All the 'well-fitted models' in segment I. We use the same symbols in this figure as in Fig. 6. It is notable that the scale of orange arrows is different from that of pink arrows. Figures (a)-(I) are rearranged in descending order of residual reductions in the fault estimation. Figure S6. All the 'well-fitted models' in segment II. We use the same symbols in this figure as in Additional file 1: Figure S5. Figures (a)-(i) are rearranged in descending order of residual reductions in the fault estimation. Figure S7. All the 'well-fitted models' in segment III. We use the same symbols in this figure as in Additional file 1: Figure S5. Figures (a)-(k) are rearranged in descending order of residual reductions in the fault estimation. Figure S8. Relation between the daily moment per unit length of the estimated fault and the average of daily tremor energy during the release period in each segment when we changed the tremor energy threshold from $0.05 \times 10^{7} \mathrm{~J}$ to $3.00 \times 10^{7} \mathrm{~J}$ every $0.05 \times 10^{7} \mathrm{~J}$. Small red, blue, green, and purple dots indicate segments I, II, III, and IV respectively. Large dots are the same in Fig. 7. Figure S9. Correlation coefficients when we changed the number of data used in the calculation in descending order 
of residual reductions in the fault estimation in each segment. Red, blue, green dots indicate segments I, II, and III, respectively.

\section{Abbreviations}

GNSS: Global Navigation Satellite System; SSE: slow slip event; GEONET: GNSS Earth Observation Network System; GSI: Geospatial Information Authority of Japan; NIED: National Research Institute for Earth Science and Disaster Resilience; ETS: episodic tremor and slip; PPP: precise point positioning; JST: Japan standard time; UTC: Coordinated Universal Time.

\section{Acknowledgements}

We used the GNSS time series data of GEONET provided by the Geospatial Information Authority of Japan and the tremor catalogs provided in the "Slow Earthquake Database" (http://www-solid.eps.s.u-tokyo.ac.jp/ sloweq/), which is supported by JSPS KAKENHI Grant Number JP16H06472 and JP17H05418 in Scientific Research on Innovative Areas, "Science of Slow Earthquakes."

\section{Authors' contributions}

MF analyzed the data and wrote the paper with the supervision of TN. TN and SM contributed to design the analysis. All authors read and approved the final manuscript.

\section{Funding}

This study was supported by JSPS KAKENHI JP16K05536 and the Ministry of Education, Culture, Sports, Science and Technology (MEXT) of Japan, under its Earthquake and Volcano Hazards Observation and Research Program.

\section{Availability of data and materials}

The GNSS raw data of GEONET are available from the Geospatial Information Authority of Japan with a registration (http://terras.gsi.go.jp/), and the tremor catalogs are available from the "Slow Earthquake Database" (http://www-solid .eps.s.u-tokyo.ac.jp/ sloweq/).

\section{Ethics approval and consent to participate}

Not applicable.

\section{Consent for publication}

Not applicable.

\section{Competing interests}

The authors declare that they have no competing interests.

\section{Author details}

1 Graduate School of Science, Kyoto University, Gokasho, Uji, Kyoto 611-0011, Japan. ${ }^{2}$ Disaster Prevention Research Institute, Kyoto University, Gokasho, Uji, Kyoto 611-0011, Japan. ${ }^{3}$ Graduate School of Science, Kyoto University, Kitashirakawa Oiwake-cho, Sakyo-ku, Kyoto 606-8502, Japan.

Received: 24 May 2019 Accepted: 22 August 2019

Published online: 06 September 2019

\section{References}

Annoura S, Obara K, Maeda T (2016) Total energy of deep low-frequency tremor in the Nankai subduction zone, southwest Japan. Geophys Res Lett 43:2562-2567. https://doi.org/10.1002/2016GL067780

Baba T, Tanioka Y, Cummins PR, Uhira K (2002) The slip distribution of the 1946 Nankai earthquake estimated from tsunami inversion using a new plate model. Phys Earth Planet Inter 132:59-73. https://doi.org/10.1016/50031 -9201(02)00044-4

DeMets C, Gordon RG, Argus DF (2010) Geologically current plate motions. Geophys J Int 181:1-80. https://doi.org/10.1111/j.1365-246X.2009.04491.x

Frank WB (2016) Slow slip hidden in the noise: the intermittence of tectonic release. Geophys Res Lett 43:10125-10133. https://doi. org/10.1002/2016gl069537

Frank WB, Rousset B, Lasserre C, Campillo M (2018) Revealing the cluster of slow transients behind a large slow slip event. Sci Adv 4:eaat0661. https:// doi.org/10.1126/sciadv.aat0661
Hiramatsu Y, Watanabe T, Obara K (2008) Deep low-frequency tremors as a proxy for slip monitoring at plate interface. Geophys Res Lett 35:L13304. https://doi.org/10.1029/2008GL034342

Hirose H, Obara K (2005) Repeating short- and long-term slow slip events with deep tremor activity around the Bungo channel region, southwest Japan. Earth Planets Space 57(10):961-972. https://doi.org/10.1186/BF03351875

Hirose H, Hirahara K, Kimata F, Fujii N, Miyazaki S (1999) A slow thrust slip event following the two 1996 Hyuganada earthquakes beneath the Bungo Channel, southwest Japan. Geophys Res Lett 26:3237-3240. https://doi. org/10.1029/1999GL010999

Hirose F, Nakajima J, Hasegawa A (2008) Three-dimensional seismic velocity structure and configuration of the Philippine Sea slab in southwestern Japan estimated by double-difference tomography. J Geophys Res 113:B09315. https://doi.org/10.1029/2007JB005274

Matsu'ura M, Hasegawa Y (1987) A maximum likelihood approach to nonlinear inversion under constraints. Phys Earth Planet Inter 47:179-187. https:// doi.org/10.1016/0031-9201(87)90076-8

Nakajima J, Hasegawa A (2007) Subduction of the Philippine Sea plate beneath southwestern Japan: slab geometry and its relationship to arc magmatism. J Geophys Res 112:B08306. https://doi.org/10.1029/2006j b004770

NIED (2009a) Short-term slow slip events with non-volcanic tremors in southwest Japan (May-October, 2008). Report of the Coordinating Committee for Earthquake Prediction 81:539-548 (in Japanese)

NIED (2009b) Short-term slow slip events with non-volcanic tremors in southwest Japan (November, 2008-April, 2009). Report of the Coordinating Committee for Earthquake Prediction 82:392-397 (in Japanese)

NIED (2010a) Short-term slow slip events with non-volcanic tremors in southwest Japan (May-November, 2009). Report of the Coordinating Committee for Earthquake Prediction 83:436-443 (in Japanese)

NIED (2010b) Short-term slow slip events with non-volcanic tremors in southwest Japan (December, 2009-April, 2010). Report of the Coordinating Committee for Earthquake Prediction 84:379-383 (in Japanese)

Nishimura T (2014) Short-term slow slip events along the Ryukyu Trench, southwestern Japan, observed by continuous GNSS. Prog Earth Planet Sci 1:22-34. https://doi.org/10.1186/s40645-014-0022-5

Nishimura T, Munekane H, Yarai H (2011) The 2011 off the Pacific coast of Tohoku Earthquake and its aftershocks observed by GEONET. Earth Planets Space 63:631-636. https://doi.org/10.5047/eps.2011.06.025

Nishimura T, Matsuzawa T, Obara K (2013) Detection of short-term slow slip events along the Nankai Trough, southwest Japan, using GNSS data. J Geophys Res Solid Earth 118:3112-3125. https://doi.org/10.1002/ jgrb.50222

Obara K (2002) Nonvolcanic deep tremor associated with subduction in southwest Japan. Science 296:1679-1681. https://doi.org/10.1126/scien ce.1070378

Obara K, Kato A (2016) Connecting slow earthquakes to huge earthquakes. Science 353(6296):253-257. https://doi.org/10.1126/science.aaf1512

Obara K, Hirose H, Yamazki F, Kasahara K (2004) Episodic slow slip events accompanied by non-volcanic tremors in southwest Japan subduction zone. Geophys Res Lett 31:L23602. https://doi.org/10.1029/2004gl020848

Okada Y (1992) Internal deformation due to shear and tensile faults in a halfspace. Bull Seismol Soc Am 82(2):1018-1040

Okada Y (1995) Simulated empirical law of coseismic crustal deformation. J Phys Earth 43:697-713

Ozawa S (2017) Long-term slow slip events along the Nankai trough subduction zone after the 2011 Tohoku earthquake in Japan. Earth Planets Space 69:56. https://doi.org/10.1186/s40623-017-0640-4

Rogers R, Dragert H (2003) Episodic tremor and slip on the Cascadia subduction zone: the chatter of silent slip. Science 300:1942-1943. https://doi. org/10.1126/science.1084783

Schwartz SY, Rokosky JM (2007) Slow slip events and seismic tremor at circum-Pacific subduction zones. Rev Geophys 45:RG3004. https://doi. org/10.1029/2006rg000208

Sekine S, Hirose H, Obara K (2010) Along-strike variations in short-term slow slip events in the southwest Japan subduction zone. J Geophys Res 115:B00A27. https://doi.org/10.1029/2008jb006059

Takagi R, Obara K, Maeda T (2016) Slow slip event within a gap between tremor and locked zones in the Nankai subduction zone. Geophys Res Lett 43:1066-1074. https://doi.org/10.1002/2015GL066987 
Wdowinski S, Bock Y, Zhang J, Fang P, Genrich J (1997) Southern California permanent GPS geodetic array: spatial filtering of daily positions for estimating coseismic and postseismic displacements induced by the 1992 Landers earthquake. J Geophys Res 102:18057-18070. https://doi. org/10.1029/97jb01378

Yabe S, Ide S (2014) Spatial distribution of seismic energy rate of tectonic tremors in subduction zones. J Geophys Res Solid Earth 119:8171-8185. https://doi.org/10.1002/2014JB011383

\section{Publisher's Note}

Springer Nature remains neutral with regard to jurisdictional claims in published maps and institutional affiliations.

\section{Submit your manuscript to a SpringerOpen ${ }^{\circ}$ journal and benefit from:}

- Convenient online submission

- Rigorous peer review

- Open access: articles freely available online

- High visibility within the field

- Retaining the copyright to your article

Submit your next manuscript at $\boldsymbol{\nabla}$ springeropen.com 